\title{
Adeno-associated virus gene therapy to the rescue for Charcot-Marie-Tooth disease type 4]
}

\author{
John Svaren \\ Department of Comparative Biosciences and Waisman Center, University of Wisconsin-Madison, Madison, Wisconsin, USA.
}

\begin{abstract}
The genetic peripheral neuropathy known as Charcot-Marie-Tooth disease type 4J (CMT4J) is caused by recessive mutations in the FIC4 gene. The transformational success of adeno-associated virus (AAV) gene therapy for spinal muscular atrophy has generated substantial interest in using this approach to create similar treatments for CMT. In this issue of the $J C I$, Presa et al. provide a preclinical demonstration of efficacy using AAV-directed gene therapy for CMT4J. The study showed a dramatic improvement in both survival and neuropathy symptoms in a severe mouse model of CMT4J after administration of AAV gene therapy at several time points. The authors' approach advances the technique for delivering treatments to individuals with CMT, for which FDA-approved therapies have not yet come to the clinic.
\end{abstract}

\section{The spectrum of Charcot-} Marie-Tooth disease

Charcot-Marie-Tooth disease (CMT) refers to genetic neuropathies affecting the PNS, which are among the most common heritable diseases of the nervous system, affecting approximately one person in 2500 . The function and preservation of the long axons in the PNS are adversely affected by mutations in a variety of genes, and collaborative efforts in the field have identified CMTassociated mutations in more than 100 genes $(1,2)$, and additional genes associated with syndromic diseases, including peripheral neuropathy (3). While axonal loss ultimately drives disease progression, CMT can be classified as demyelinating or axonal. Demyelinating (CMT1) affects Schwann cell myelin and nerve conduction velocity, contrasting with axonal (CMT2), which is often characterized by primary effects on axonal transmission of motor neurons to muscle, as measured by compound muscle action potentials. CMT1/2 designations generally reflect dominant disorders, but CMT4 classifications such as CMT type 4J (CMT4J) refer to recessive inheritance.

Given the diversity of genetic causes, adeno-associated virus-mediated (AAVmediated) gene-replacement therapy has emerged as an inherently flexible approach that researchers can customize to broadly apply across the spectrum of CMT. Indeed, clinical trials have already commenced for giant axonal neuropathy (GAN) (4), a rare disorder that can be considered a CMT neuropathy. As a result of the cumulative efforts of many laboratories and companies, the safety studies of AAV delivery to motor neurons have provided additional confidence in this approach, and the regulatory path to FDA approval is well mapped (5). It will take some time to fully evaluate the long-term persistence of therapeutic gene expression within AAV-treated individuals, but the single administration of a therapeutic agent is a big advantage of this approach.

Delated Article: https://doi.org/10.1172/JCI137159

Conflict of interest: JS has a patent titled "Method to predict likelihood of inherited peripheral neuropathy in mammals" (P180279US02) and a pending patent titled "Regulatory elements for Schwann cell-specific gene expression" (P200262US01)

Copyright: @ 2021, American Society for Clinical Investigation.

Reference information: J Clin Invest. 2021;131(11):e149492. https://doi.org/10.1172/JCI149492.

Functional studies of FIC4 in phosphoinositide metabolism

The recessive CMT4 disorders are an attractive gene-therapy target due to the loss-of-function mutations that suggest minimal interference with therapeutic gene delivery (6). Further, the sophisticated mechanistic and mouse model studies from the Meisler laboratory and others in the field indicate that CMT4J is an optimal target (7-12). The FIG4 gene codes for a phosphatase involved in phosphatidyl inositol $(3,5) \mathrm{P} 2$ (PI[3,5]P2) metabolism, which is affected in several other types of CMT neuropathy (Figure 1). While FIG4 is expressed in many cell types, the clinical manifestations of CMT4J are most prominently displayed in the peripheral nerve. Interestingly, patients with CMT4J are typically compound heterozygotes with one null allele and a missense allele (often I41T) that destabilizes the mutant FIG4 protein (10). Homozygous null alleles of FIG4 cause the more severe Yunis-Varon syndrome (7), involving skeletal abnormalities and more profound CNS deficits. FIG4 recessive mutations have also been associated with epilepsy with CNS hypomyelination (13). Mutations in MTMR genes (Figure 1A; MTMR2, MTMR13, MTMR5) are classified as CMT4B1, CMT4B2, and CMT4B3, and genetic interactions of FIG4 and MTMR2 have been demonstrated in mouse models (14).

FIG4 exists within a complex with the VAC14 scaffolding protein and the lipid 5 kinase PIKFYVE, involved in PI $(3,5) \mathrm{P} 2$ and PI(5)P formation. PI $(3,5) \mathrm{P} 2$ has been implicated in the autophagy pathway and lysosomal function, and its study has revealed a number of important mechanistic points. FIG4 mutant cells exhibit extensive vacuolation and have impaired lysosomal fission, but normal fusion. FIG4 interacts with TRIPML1 channels and dynamin1, involved in lysosomal fission (15). Intriguingly, a mutant FIG4 lacking phosphatase activity can nonetheless rescue vacuolation in cultured CMT4J 
A
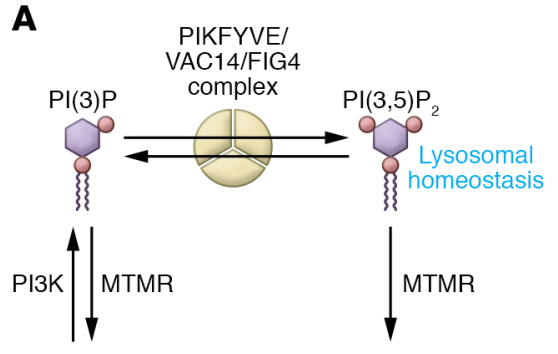

$\mathrm{Pl}$

8

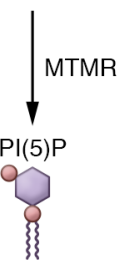

B
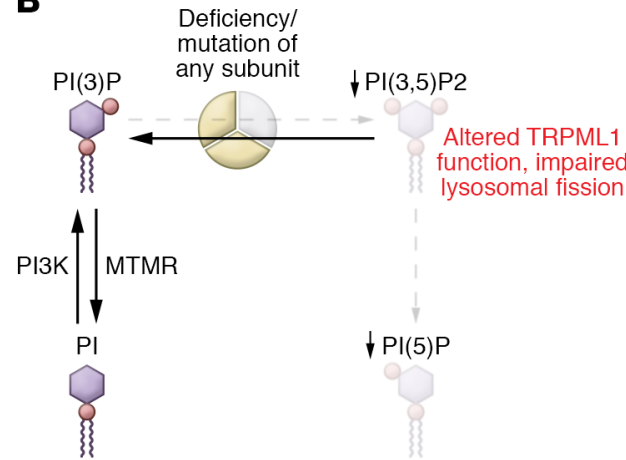

ysosomal fission

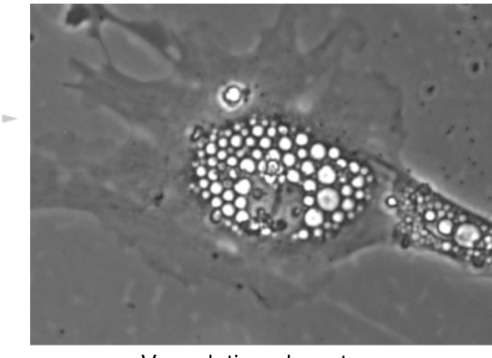

Vacuolation phenotype

Figure 1. Loss of FIG4 depletes PI(3,5)P2 and PI(5)P and causes CMT neuropathy. (A) Although FIG4 is a lipid phosphatase that can convert PI(3,5)P2 to $\mathrm{PI}(3) \mathrm{P}$, it exists within a complex with the lipid 5 kinase (PIKFYVE) and VAC14. Deficiency of any of the subunits results in deficiency of PI(3,5)P2 and $\mathrm{PI}(5) \mathrm{P}$, which are required for lysosomal homeostasis. (B) The altered TRIPML1 channel function and impaired lysosomal fission likely contribute to the prominent vacuolation phenotype in CMT4J. Similarly, cultured embryonic fibroblasts from Fig4 null mice accumulate large, acidic vacuoles (photograph provided with permission by G. Lenk and M. Meisler).

fibroblasts and partially extend life span in vivo (11), indicating that FIG4 is most critically required for the integrity of the protein complex and its PIKFYVE kinase activity. Accordingly, analysis of primary fibroblasts from patients with CMT4J showed reductions in PI(5)P and PI(3,5) P2 (16). Importantly, the role of FIG4 is not restricted to development, as induced deletion of the gene in adult mice provokes many facets of the disease (17).

\section{Identifying target cell types for FIC4 restoration}

As a potentially confounding issue, patients with CMT4J have slowed nerve-conduction velocities consistent with demyelination, indicating that Schwann cells are involved (18). However, elegant mouse studies with conditional and null mutants indicate that a specific neuronal knockout causes ataxia, tremor, and hypomyelination of peripheral nerve, but prolongs survival compared with the null. In addition, expressing FIG4 specifically in neurons can rescue the hypomyelination and early lethality/ataxia of the null mouse (9). Therefore, neuronspecific deficits underlie the most severe aspects of CMT4J. Nonetheless, the myelin defects in Fig4 null mice reflect to some extent a Schwann cell-intrinsic function, as there are clear myelin development deficits, myelin degeneration, and later axonal loss with a Schwann cellspecific deletion of Fig4 $(12,18)$, although segmental demyelination only occurs with complete Fig4 null alleles. These studies illustrate the mutually dependent nature of axon and Schwann cell development and maintenance.

\section{AAV-mediated rescue of FIG4 function}

With the CMT4J animal model and mechanistic studies, the stage was set to test the now well-established gene-therapy approaches employing CNS routes of AAV delivery, as used in spinal muscular atrophy (SMA) and GAN $(4,5)$. In this issue of the JCI, Presa et al. (19) report on their performance of a preclinical study using a spontaneous null mutant in Fig4, identified as the pale tremor mouse $(p l t)$, which tends to model the more severe Yunis-Varon phenotype, with a median survival of only a few weeks (8). However, despite the severity of this model, AAV administration of human FIG4 expressed from a ubiquitous promoter in one-dayold plt mice dramatically restored survival beyond 12 months of age, albeit with lower body weights. Importantly, behavioral tests showed complete rescue of motor deficits. Since FIG4 affects phosphoinositide metabolism, overexpression could adversely affect the balance of the various phosphoinostides (Figure 1), but the metabolic impact is likely limited by the endogenous levels of VAC14 and PIKFYVE, and Presa et al. (19) showed that AAV-mediated expression of FIG4 in wild-type mice did not appear to cause any issues.

Another factor for consideration is how soon therapeutic gene delivery must occur to rescue function, as gene therapy has much less efficacy after a certain age in children with severe forms of SMA (5). While the presentation of CMT4J is generally less severe than that of the most severe form of SMA (type-I), there is a spectrum of early onset to adult-onset disease (13, 18). To address this issue, injections were also done at $\mathrm{P} 4$, and most mice also survived up to one year. Intrathecal injections prolonged survival to a lesser degree (90 days) when performed at P7, but no increased survival was evident with P11 injections. Nonetheless, motor function was dramatically improved with the P7 injections. While the plt model is a severe model of CMT4J, it remains to be seen how much the neuropathy will improve (or at least stabilize) in human trials, which will likely depend upon the degree of axon loss prior to AAV administration. These data clearly justify a gene-therapy approach to CMT4J, but limiting expression to neurons may also limit long-term functional recovery $(12,18)$. In Presa et al. (19), nerve conduction velocities and myelin thickness were restored to normal in the early treatment group even though less than $10 \%$ of Schwann cells expressed the AAV-borne human FIG4. However, the extent of recovery in human trials is difficult to predict from preclinical studies.

The studies described in Presa et al. (19) represent a major breakthrough in extending the success of gene therapy for SMA to not only CMT4J, but also to other types of CMT. The prospects of small molecule development for rare forms of CMT such as CMT4J can be daunting, so 
the gene-therapy options are likely the most rapid path to clinic. In some respects, mildly progressing symptoms in CMT compared with severe forms of SMA may portend even greater treatment efficacy in the spectrum of CMT neuropathies, although treatment success will likely vary in different subtypes of CMT. In addition, most types of CMT result from dominant mutations, so strategic adaptations may need to be employed (6). Another major goal in the field is to expand similar AAV technology to target demyelinating neuropathy, which will require efficient delivery to Schwann cells (20). Advances in this targeting endeavor will enable application of similar gene-therapy approaches to most types of CMT.

\section{Acknowledgments}

I thank Miriam Meisler, Jun Li, and Alessandra Bolino for helpful discussions in preparing this article.

Address correspondence to: John Svaren, Department of Comparative Biosciences and Waisman Center, University of Wisconsin-Madison, 1500 Highland Ave., Madison, Wisconsin 53705, USA. Email: john.svaren@wisc.edu.
1. Timmerman V, et al. Genetics of CharcotMarie-Tooth (CMT) disease within the frame of the human genome project success. Genes (Basel). 2014;5(1):13-32.

2. Pipis $M$, et al. Next-generation sequencing in Charcot-Marie-Tooth disease: opportunities and challenges. Nat Rev Neurol. 2019;15(11):644-656.

3. Rossor AM, et al. Peripheral neuropathy in complex inherited diseases: an approach to diagnosis. J Neurol Neurosurg Psychiatry. 2017;88(10):846-863.

4. Bailey RM, et al. Development of intrathecal AAV9 gene therapy for giant axonal neuropathy. Mol Ther Methods Clin Dev. 2018;9:160-171.

5. Ravi B, et al. Gene-targeting therapeutics for neurological disease: lessons learned from spinal muscular atrophy. Annu Rev Med. 2021;72:1-14.

6. Morelli KH, et al. Gene therapies for axonal neuropathies: Available strategies, successes to date, and what to target next. Brain Res. 2020;1732:146683.

7. Campeau PM, et al. Yunis-Varón syndrome is caused by mutations in FIG4, encoding a phosphoinositide phosphatase. Am J Hum Genet. 2013;92(5):781-791.

8. Chow CY, et al. Mutation of FIG4 causes neurodegeneration in the pale tremor mouse and patients with CMT4J. Nature. 2007;448(7149):68-72.

9. Ferguson CJ, et al. Neuronal expression of Fig4 is both necessary and sufficient to prevent spongiform neurodegeneration. Hum Mol Genet. 2012;21(16):3525-3534.

10. Lenk GM, et al. Pathogenic mechanism of the FIG4 mutation responsible for Charcot-Marie-Tooth disease CMT4J. PLoS Genet. 2011;7(6):e1002104.
11. Lenk GM, et al. Rescue of neurodegeneration in the Fig4 null mouse by a catalytically inactive FIG4 transgene. Hum Mol Genet. 2016;25(2):340-347.

12. Vaccari I, et al. Loss of Fig4 in both Schwann cells and motor neurons contributes to CMT4J neuropathy. Hum Mol Genet. 2015;24(2):383-396.

13. Nicholson G, et al. Distinctive genetic and clinical features of CMT4J: a severe neuropathy caused by mutations in the $\mathrm{PI}(3,5) \mathrm{P}_{2}$ phosphatase FIG4. Brain. 2011;134(pt 7):1959-1971.

14. Vaccari I, et al. Genetic interaction between MTMR2 and FIG4 phospholipid phosphatases involved in Charcot-Marie-Tooth neuropathies. PLoS Genet. 2011;7(10):e1002319.

15. Zou J, et al. Reactivation of lysosomal $\mathrm{Ca} 2+$ efflux rescues abnormal lysosomal storage in FIG4-deficient cells. J Neurosci. 2015;35(17):6801-6812.

16. Shisheva A, et al. Severe consequences of SAC3/FIG4 phosphatase deficiency to phosphoinositides in patients with Charcot-Marie-Tooth disease type-4J. Mol Neurobiol. 2019;56(12):8656-8667.

17. Mironova YA, et al. Protective role of the lipid phosphatase Fig4 in the adult nervous system. Hum Mol Genet. 2018;27(14):2443-2453.

18. Hu B, et al. Myelin abnormality in CharcotMarie-Tooth type $4 \mathrm{~J}$ recapitulates features of acquired demyelination. Ann Neurol. 2018;83(4):756-770.

19. Presa M, et al. AAV9-mediated FIG4 delivery prolongs life span in Charcot-Marie-Tooth disease type 4J mouse model. J Clin Invest. 2021;131(11):e137159.

20. Sargiannidou I, et al. Gene therapy approaches targeting Schwann cells for demyelinating neuropathies. Brain Res. 2020;1728:146572. 